\title{
Association of meningioma and intracranial aneurysm: Report of five cases and review of literature
}

\author{
Vijayakumar Javalkar, Bharat Guthikonda, Prasad Vannemreddy, Anil Nanda \\ Department of Neurosurgery, Louisiana State University Health Sciences Center, Shreveport, LA, USA
}

\author{
Address for correspondence: \\ Dr. Anil Nanda, \\ Department of Neurosurgery, \\ Louisiana State University Health \\ Sciences Center - Shreveport, \\ 1501 Kings Highway, \\ Shreveport, LA 71130 , USA. \\ E-mail: ananda@Isuhsc.edu
}

DOI: $10.4103 / 0028-3886.59475$

\begin{abstract}
Meningiomas associated with intracranial aneurysms are very rare. The co-existence of both lesions is not only a diagnostic challenge but also has important therapeutic implications. We analyzed our experience of five such patients, the second largest group in the literature. All the lesions were successfully managed surgically. All the patients were female. The location of the meningioma was clinoidal (2), planum sphenoidale (1), petroclival (I) and pterional (I). Three patients had posterior communicating (PCOM) artery aneurysm associated with meningioma. The other aneurysms were internal carotid artery (ICA) and anterior communicating artery (ACOM). One patient with pterional meningioma had an anomalous meningeal artery arising from the right ophthalmic artery. In three patients both lesions underwent treatment at the same sitting. In two patients meningioma underwent excision first followed by aneurysm clipping. From our experience the incidence of meningiomas associated with aneurysms was $1.1 \%$, which is not higher than the incidence of aneurysms in the general population.
\end{abstract}

Key words: Aneurysm, endovascular, meningioma, surgery

\section{Introduction}

Few case reports are published in the literature documenting the association between brain tumors and aneurysms. ${ }^{[1-3]}$ The incidence is estimated to be between $0.3-0.7 \% .^{[2,3]}$ Of all the brain tumors, meningioma is the commonest tumor showing such an association. Co-existence of both lesions is not only a diagnostic challenge but also has important therapeutic implications. In this paper we present five such cases. Our series of five cases constitute the second largest group.

\section{Materials and Methods}

We retrospectively reviewed the medical records of all the patients with meningiomas who underwent surgical excision to look for the cases with an associated aneurysm. In our series of 426 cranial meningiomas only five [Table 1] were associated with aneurysms $(1.1 \%)$.

\section{Case 1}

A 70-year-old lady presented with sudden severe headache and diminished vision in the right eye. On examination she had right third nerve palsy with neck stiffness. CT scan [Figure 1] revealed right pterional meningioma. Angiogram revealed characteristic blush of meningioma, anomalous meningeal artery arising from right ophthalmic artery and PCOM aneurysm [Figure 2a,

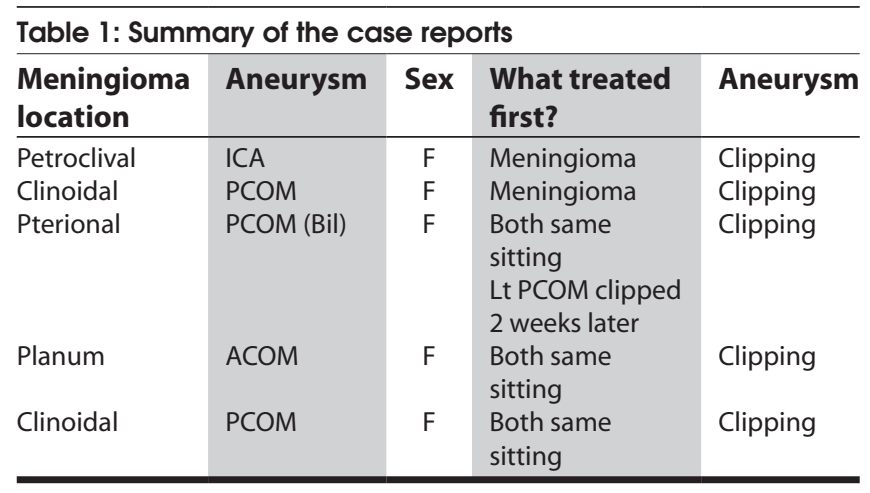




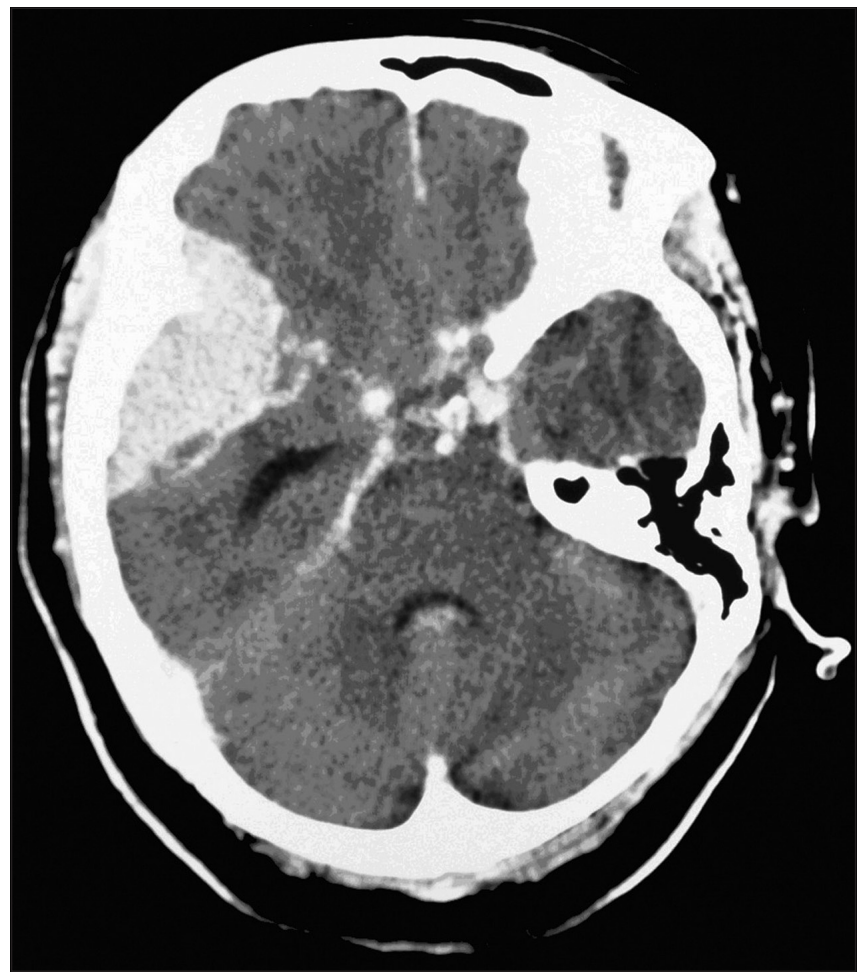

Figure 1: CT scan showing pterional meningioma (case 1)

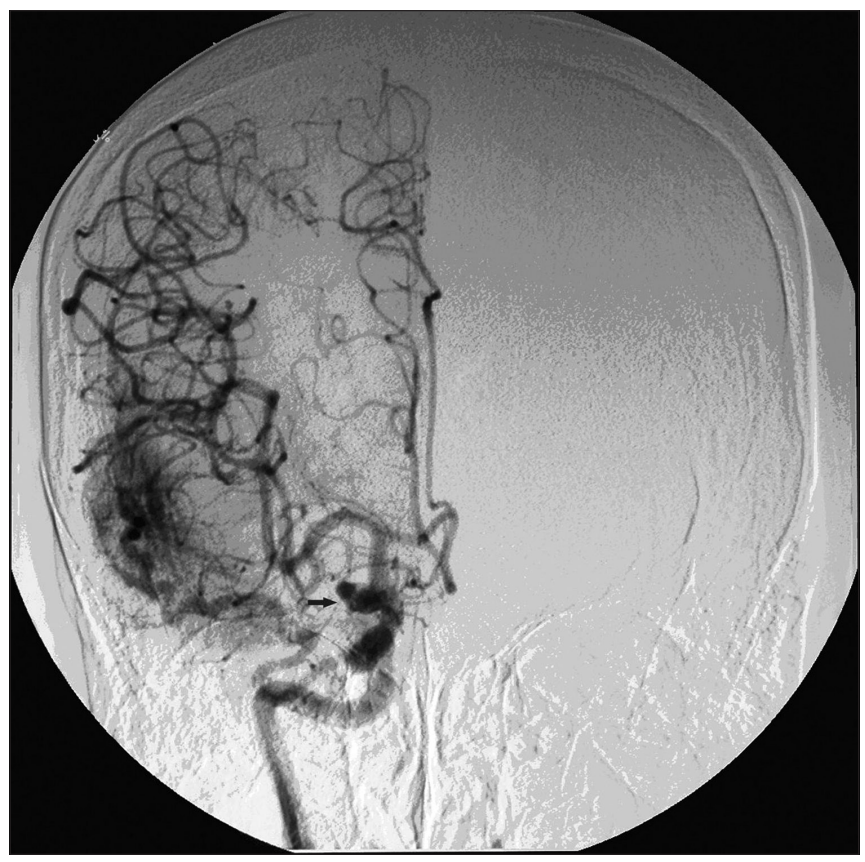

Figure 3: Angiogram showing posterior communicating aneurysm (case 1)

$2 \mathrm{~b}$ and 3$]$. She underwent excision of meningioma and clipping of the right PCOM aneurysm initially followed by clipping of the left PCOM aneurysm two weeks later. Patient did well after surgery.

\section{Case 2}

The patient, a 55-year-old lady, initially underwent

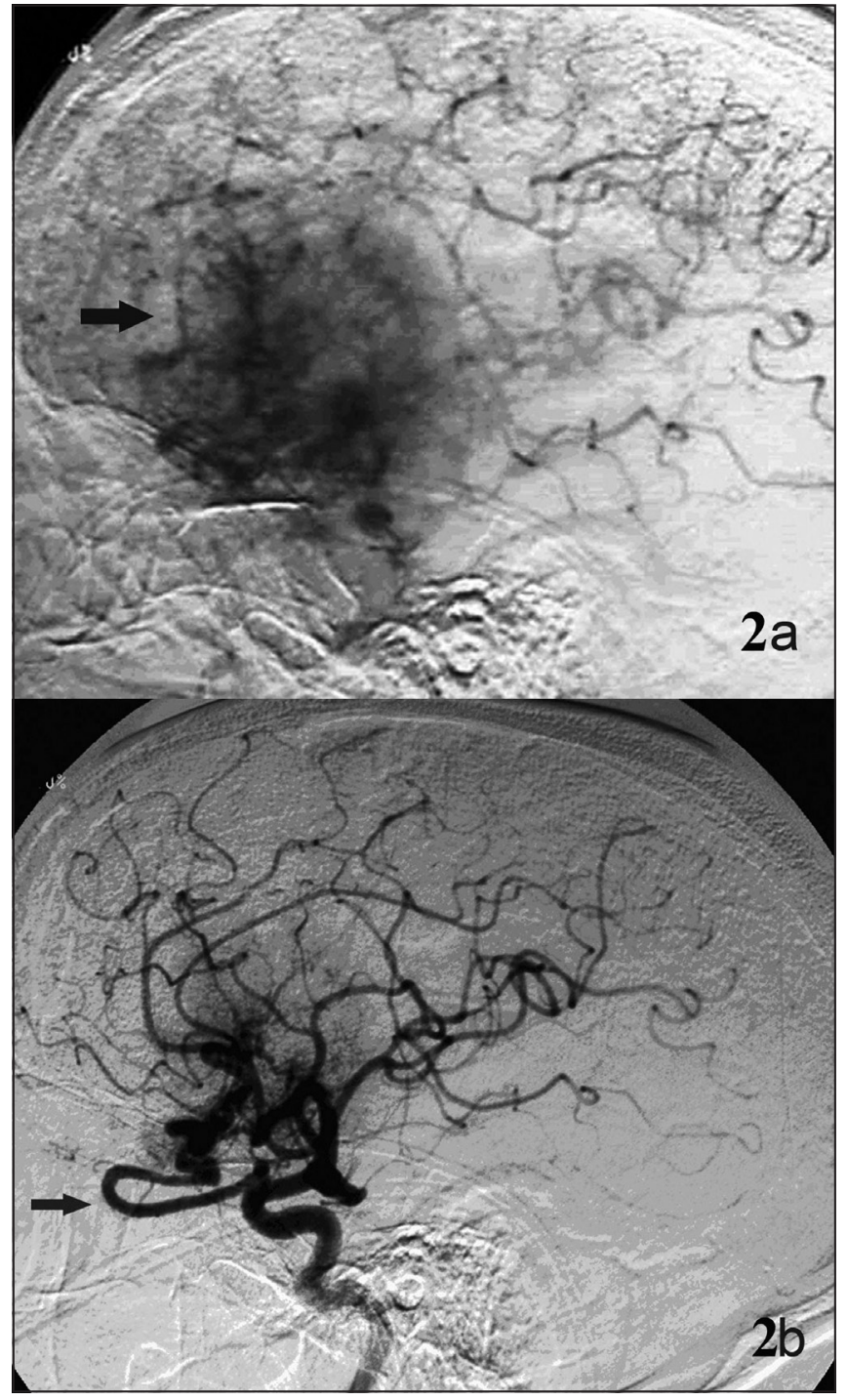

Figure 2: Angiogram showing characteristic blush of meningioma (2a) and anomalous meningeal artery arising from hypertrophied right ophthalmic artery $(2 \mathrm{~b})$ (case 1)

surgical excision for clinoidal meningioma in 1995. Excision was total. She had regular follow-up with MRI scans, which did not reveal any residual or recurrence. In 2006 she was evaluated for speech disturbance with MRI scan which revealed an aneurysm. MRI and MRA images [Figures 4 and 5] suggested Right PCOM aneurysm. She underwent successful clipping of the unruptured aneurysm. Patient is doing well after surgery.

\section{Case 3}

This patient is a 37 year-old-lady who had a left petroclival meningioma resected in two stages in 1999. She also had an incidental right internal carotid artery (ICA) aneurysm, clipped subsequently. In 2002, she underwent gamma-knife radiosurgery for the residual meningioma. Presently she is doing very well. Figure 6 shows the residual Petroclival meningioma. Figure 7 shows the clip artifact. 


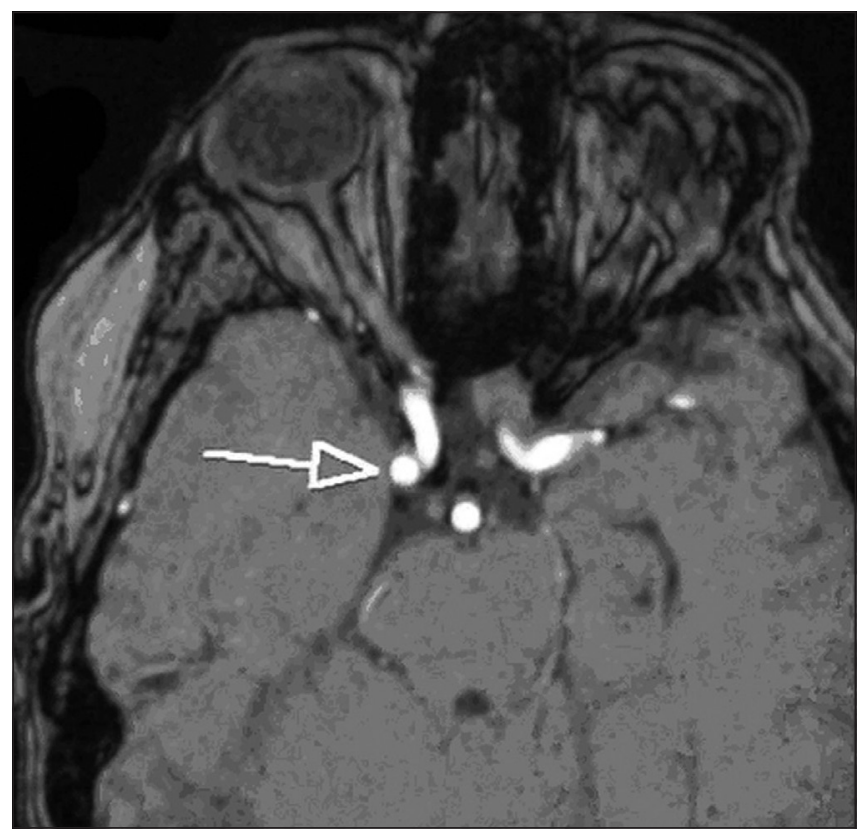

Figure 4: MRI showing posterior communicating aneurysm (case 2)

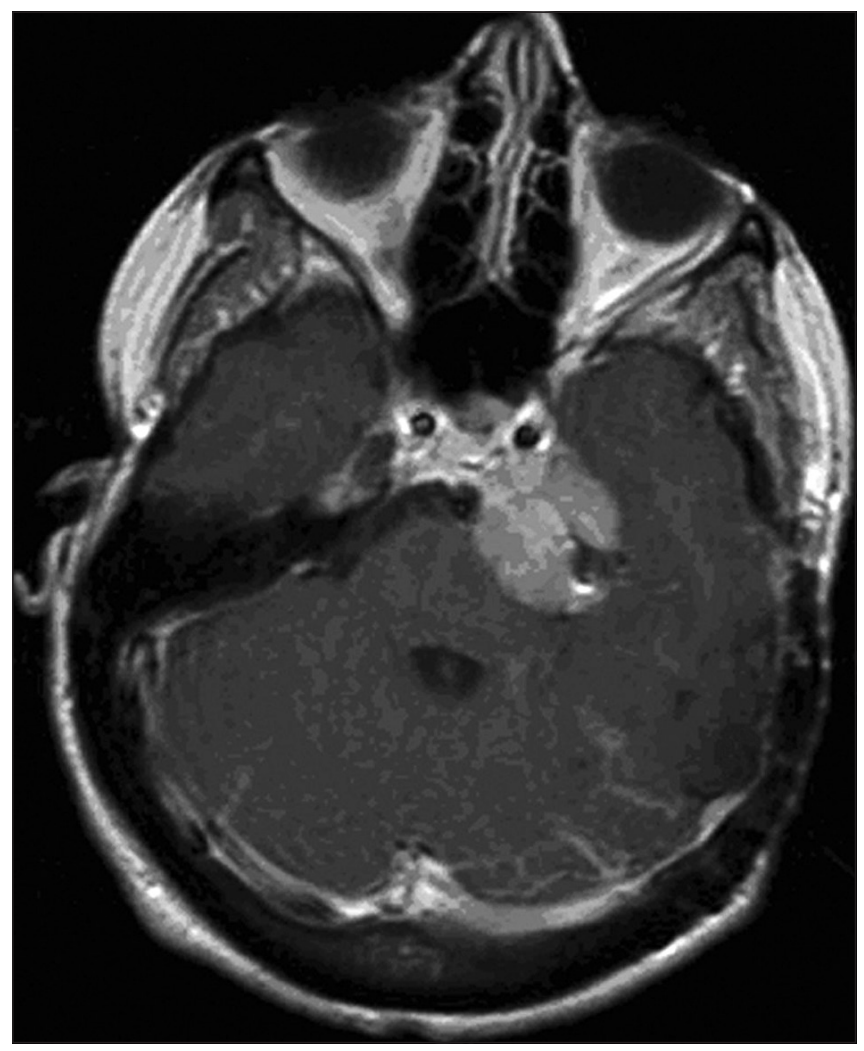

Figure 6: Residual petroclival meningioma (case 3)

\section{Case 4}

A 63 year- old-lady suffered the worst headache of her life. She subsequently deteriorated to a Glasgow coma scale (GCS) score 8 and was intubated. CT showed subarchnoid hemorrhage (SAH) and angiogram showed anterior communicating (ACOM) artery aneurysm. Patient underwent clipping of the aneurysm. The planum

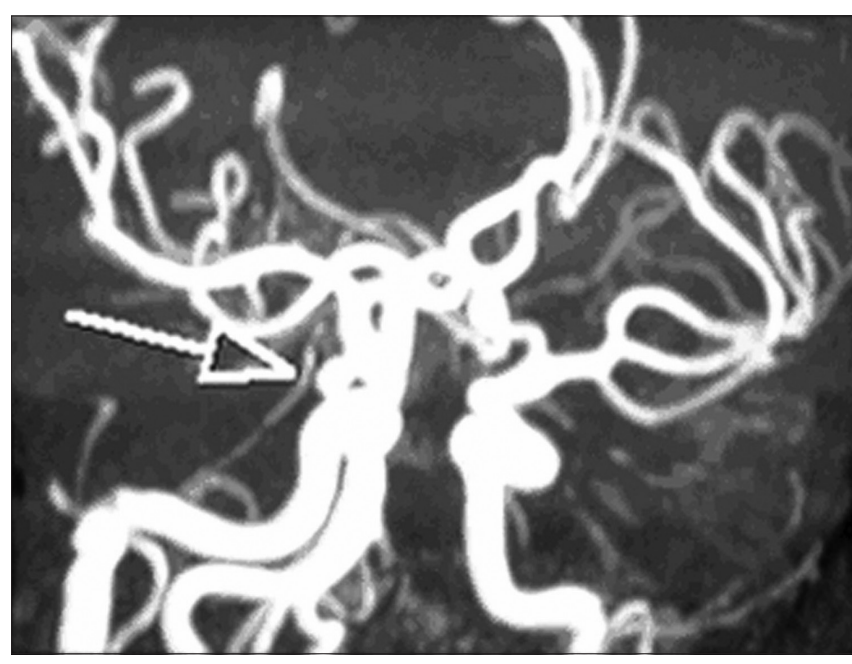

Figure 5: MRA showing the posterior communicating aneurysm (case 2)

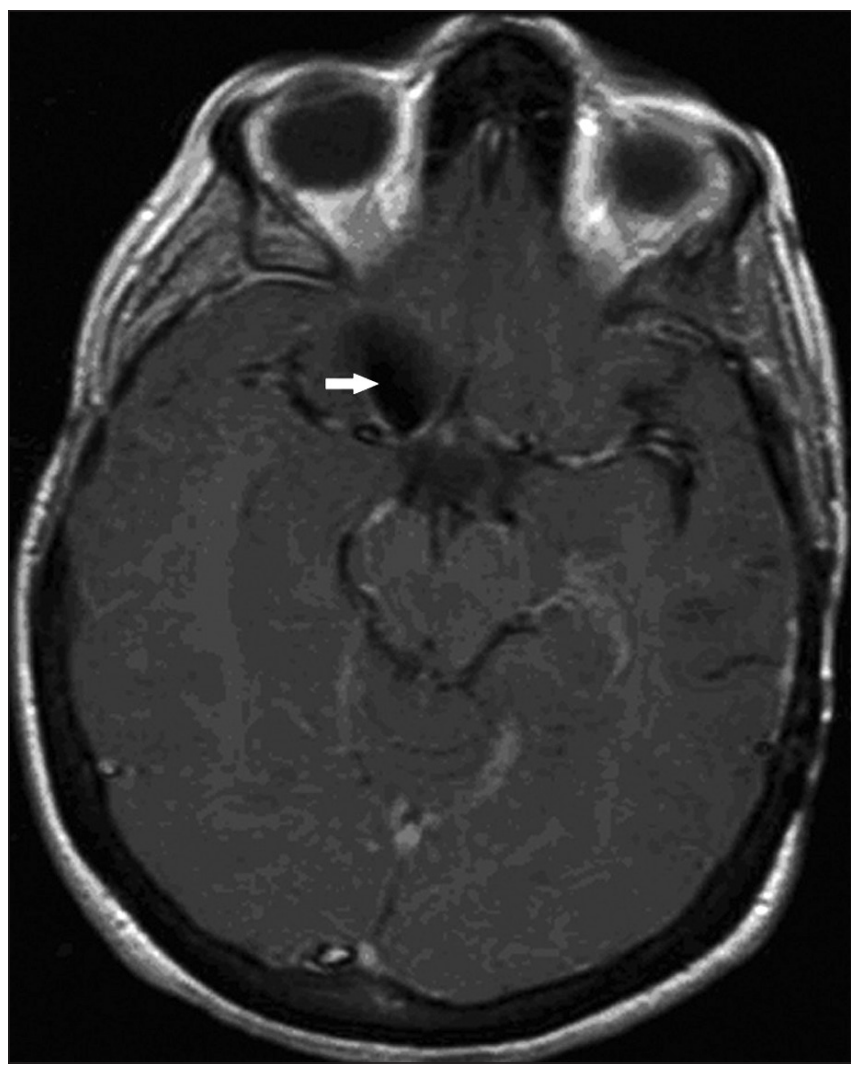

Figure 7: MRI showing clip artifact on the right side (case 3)

sphenoidale meningioma which was small was excised. Intra-operative angiogram showed successful clipping of the aneurysm. The meningioma was small and it was appreciated only during surgery.

\section{Case 5}

The patient, a 61-year-old, lady presented with sudden severe headache. CTA (CT angiogram) and angiogram showed a large PCOM aneurysm [Figure 8]. Endovascular embolization was attempted but not successful. Patient subsequently underwent surgical clipping. During 
operation there was an abnormal looking area lateral to the optic nerve. The mass had pushed the carotid away and was measuring $2 \times 1.5 \mathrm{~cm}$ in size [Figure 9]. It was resected totally followed by aneurysm clipping. Histopathology was consistent with meningioma.

\section{Discussion}

The association between intracranial meningioma and aneurysm was first reported by Arieti et al. ${ }^{[4]}$ in 1944. Licata et al. ${ }^{[1]}$ contributed eight cases, followed by our series. Numerous hypotheses were put forward to support the co-existence of meningiomas and
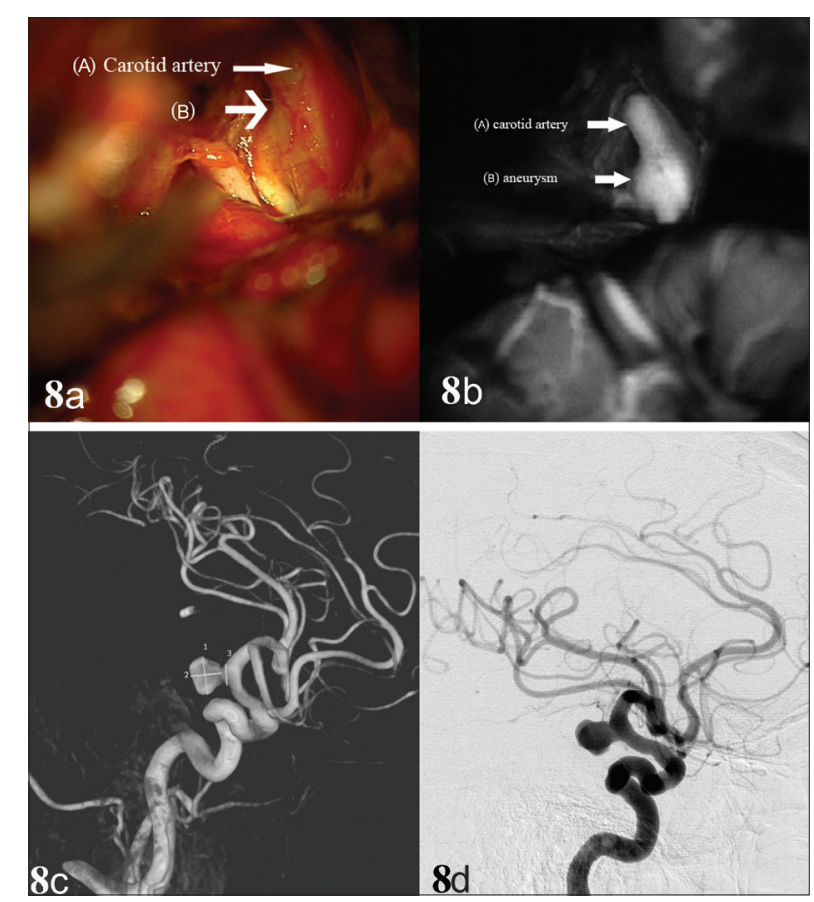

Figure 8: Intraoperative photograph (a), Indocyanine green angiogram (b), CT angiogram (c) and four vessel angiogram (d) showing posterior communicating aneurysm (case 5). Arrow (A) points to carotid artery. Arrow (b) points to the aneurysm in Figure $a$ and $b$

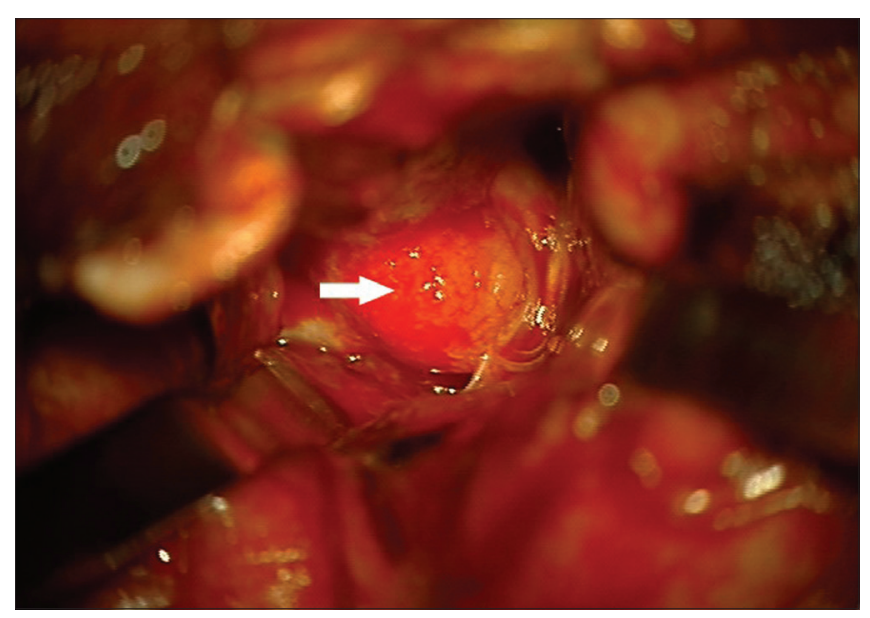

Figure 9: Intraoperative picture showing clinoidal meningioma (case 5) aneurysms. The increased regional blood flow associated with vascular meningiomas might explain a subset of aneurysms like those present on the feeding arteries. ${ }^{[3]}$ This hypothesis cannot explain contra lateral aneurysms. In the case described by Kandel et al. ${ }^{[5]}$ a typical MCA aneurysm was associated with fronto temporal meningioma. The authors attributed the formation of the aneurysm to the damage that the meningioma caused to the wall of the artery based on tumor adhesion to the arterial adventitia. Pia et al. ${ }^{[3]}$ had proposed that a dysgenetic factor might explain a high incidence of meningiomas associated with aneurysms. Co-existence of cerebral aneurysms and meningiomas has important implications in both clinical diagnosis and treatment. The important question is whether angiogram is required in all patients with meningiomas? To answer this one needs to know the incidence of such an association. In the study by Handa et al. ${ }^{[2]}$ out of 956 patients with brain tumors, only 7 had an associated aneurysm $(0.7 \%)$, out of which only 1 case was meningioma. Taylor ${ }^{[6]}$ in his series of 1500 cases of verifiable primary intracranial tumors could find only five tumors with aneurysms $(0.3 \%)$, out of which two were menigiomas. Handa et al. ${ }^{[2]}$ in his analysis had documented that out of 134 tumors with aneurysms, 40 were meningiomas (30\%). In both these large series there was no split up of brain tumors into various pathologies; hence exact incidence of meningiomas with aneurysms could not be calculated.

In our series of 426 intracranial meningiomas only five were associated with aneurysms (1.1\%). Larger series are essential in order to calculate the true incidence. This incidence might still be lower since not all patients with menigiomas undergo routine angiogram. The estimated incidence from our experience $(1.1 \%)$ was not higher than the incidence of aneurysms in the general population and this probably suggests that the co-existence of these two conditions may be due to chance alone. In the literature there was only one report of genetic disorder showing such association (Klippel-Trenaunay syndrome with multiple meningiomas and arterial aneurysms). ${ }^{[7]}$ Klippel-Trenaunay syndrome is a rare neurocutaneous disorder in which skeletal hypertrophy, vascular nevi and vessel anomalies coexist. In this literature analysis there was insufficient evidence of dysgenetic hypothesis as proposed by Pia. ${ }^{[3]}$ With such a low incidence, routine angiogram to pick up aneurysms is not indicated in each and every patient with meningiomas. However, patients with meningiomas undergo angiograms due to other reasons like tumor embolization, to know the relationship with major vessels and tumor encasement of major vessels. Alternatively, all patients with meningiomas can undergo MRA and CT angiograms which are non-invasive to pick up any incidental aneurysms. 
Various treatment options are available including conservative management, treating both pathologies simultaneously or sequentially. If the aneurysm is in close proximity to the tumor and if the surgeon can access it then it can be clipped in the same sitting. In the series published by $\mathrm{Pia},{ }^{[3]} 21$ patients with benign tumors (14 meningiomas and 7 pituitary adenomas) were treated at the same time. Sometimes aneurysm may be intratumoral. ${ }^{[5,8,9]}$ The other option that is available is coil embolization of the aneurysms. $\mathrm{O}^{\prime}$ Neill[10] published the first report where the middle meningeal artery was occluded proximal to the aneurysm with platinum coils followed by meningioma excision. In a similar case described by Lama et al. ${ }^{[11]}$ embolization of the aneurysm was performed preoperatively with platinum coils followed by surgical excision of meningioma. Similar approach had been used by Javadpour et al..$^{[12]}$ Alternatively incidental aneurysms can be managed conservatively but it is difficult to predict the effects of hemodynamics associated with meningiomas especially if they are ipsilateral to the tumor and the effect of craniotomy is also difficult to predict. For these aneurysms it is more appropriate if they are dealt with either surgery or endovascular techniques.

\section{Conclusion}

From our experience only $1.1 \%$ of meningiomas had an associated aneurysm. This incidence is less than the incidence of aneurysm in the general population. Even though the underlying mechanisms for such an association are not clear, with such a low incidence, it might be due to chance alone. If the aneurysm is ipsilateral and in close proximity to meningioma, both lesions can be managed simultaneously or aneurysm can be treated by endovascular coiling prior to craniotomy for meningioma to make surgery on the meningioma safer. If the aneurysm is located contra lateral to the side of meningioma or cannot be approached at the time of meningioma excision, they can be dealt with by either surgery or endovascular procedures.

\section{References}

1. Licata C, Pasqualin A, Freschini A, Barone G, Da Pian R. Management of associated primary cerebral neoplasm's and vascular malformations. Acta Neurochir 1986;82:28-38.

2. Handa J, Matsuda I, Handa H. Association of brain tumor and intracranial aneurysms. Surg Neurol 1976;6:25-9.

3. Pia HW, Obrador S, Martin JG. Association of brain tumours and arterial intracranial aneurysms. Acta Neurochir (Wien) 1972;27:189-204.

4. Arieti S. Multiple meningioma and meningiomas associated with other brain tumors. J Neuropath Exp Neurol 1944;3:255-70.

5. Kandel E, Ludkovskaya I, Dobjansky N. Aneurysm inside meningioma. Case report. Acta Neurochir (Wien) 1986;81:72-6.

6. Taylor PE. Delayed postoperative hemorrhage from intracranial aneurysm after craniotomy for tumour. Neurol 1961;11;225-31.

7. Spallone A, Tcherekayev VA. Simultaneous occurrence of aneurysm and multiple meningioma in Klippel-Trenaunay patients: Case report. Surg Neurol 1996;45:241-4.

8. Tancioni F, Egitto MG, Tartara F. Aneurysm occurring within a meningioma: Case report. Br J Neurosurg 1998;12:588-91.

9. Cha KY, Park SK, Hwang YS, Kim TH. Strategy for the patient with Tuberculum sellae meningioma combining bilateral internal artery aneurysm. J Korean Neurosurg Soc 2005;38:151-4.

10. O'Neill OR, Barnwell SL, Silver DJ. Middle meningeal artery aneurysm associated with meningioma: Case report. Neurosurgery 1995;36:396-8.

11. Lama M, Mottolese C. Middle meningeal artery aneurysm associated with meningioma. J Neurosurg Sci 2000;44:39-41.

12. Javadpour M, Khan AD, Jenkinson MD, Foy PM, Nahser HC. Cerebral aneurysm associated with an intracranial tumour: Staged endovascular and surgical treatment in two cases. Br J Neurosurg 2004;18:280-4.

Accepted on 02-11-2009

Source of Support: Nil, Conflict of Interest: None declared. 